\title{
Fabrication of site-controlled InGaN quantum dots using reactive-ion etching
}

\author{
Leung Kway Lee and P.-C. Ku* \\ Department of Electrical Engineering and Computer Science, University of Michigan, 1301 Beal Ave., Ann Arbor, MI 48109, USA
}

Received 8 July 2011, revised 17 August 2011, accepted 26 August 2011

Published online 9 December 2011

Keywords quantum dots, indium gallium nitride, reactive-ion etching

* Corresponding author: e-mail peicheng@umich.edu, Phone: +1 (734) 764-7134

We adopted the simple top-down etching to fabricate site- and dimension-controlled InGaN quantum dots. Each quantum dot is disk shaped and embedded in a nanoscale pillar. Arrays of nanopillars with varying densities and nanopillar diameters were fabricated from an InGaN/GaN single quantum well using inductivelycoupled plasma reactive-ion etching. Micro-photoluminescence $(\mu-\mathrm{PL})$ was used to characterize the emis-

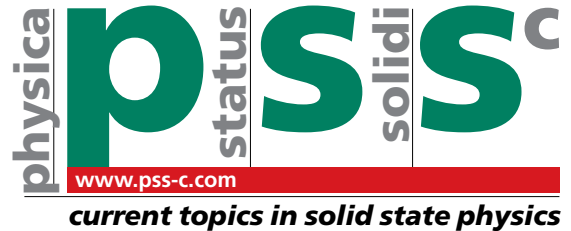

current topics in solid state physics sion properties of individual and ensemble of nanopillars. Strong and distinct PL signal of a single nanopillar was observed even at the room temperature. The emission was found to exhibit characteristics from a discrete energy state that is homogeneously broadened. The ensemble of nanopillars exhibited a similar emission linewidth as the single nanopillar, indicating a well controlled quantum dot dimensions and uniformity.
1 Introduction III-nitride semiconductor quantum dots (QDs) exhibit large exciton binding energy $(\geq 26$ $\mathrm{meV})[1,2]$ and are ideal candidates to exploit various quantum optical phenomena at high temperatures including single-photon emission [3], strong-coupling [4, 5], indistinguishable photon generation [6] and one-atom lasing [7]. The practical use of the III-nitride QDs as quantum light sources often requires the addressability of a single QD. For example, strong-coupling requires the precise placement of a single QD at the anti-node of an optical cavity. Commonly, QDs are fabricated by the strain-induced Stranski-Krastanow (SK) growth mode which does not enforce control over the dot's site or dimension, making them difficult to be utilized on the device level. To provide siteand dimension-control to individual dots, one may apply selective area epitaxy (SAE), also known as the patterned growth [8], or the top-down approach i.e. to etch a single quantum well (SQW) into nanopillars (or nanoposts). For $\mathrm{SAE}$, the site, dimension and material quality of the asgrown nanostructures are very sensitive to the growth condition as the growth mechanism of a patterned substrate varies significantly from an unpatterned one [8-10]. The top-down approach, on the other hand, utilizes the matured epitaxial techniques and nanolithography and serves as a more reliable method for making controlled nanostructures. Previously, the top-down approach has been used by several research groups to produce site-controlled quantum dots in III-V materials $[11,12]$. For III-nitride material systems, however, studies often focused on nanopillars made from multiple-quantum-wells (MQWs) [13-17]. To date, high-quality, site-controlled III-nitride quantum dots fabricated using the top-down approach have not been realized. In this paper, we demonstrate a viable fabrication method to produce site-controlled InGaN quantum dots exhibiting extremely good uniformity and room-temperature (RT) single-dot luminescence.

\section{Sample preparation and experimental setup}

The sample preparation is illustrated in Fig. 1(a). First, a $1.5-\mu \mathrm{m}$ thick gallium nitride $(\mathrm{GaN})$ template was epitaxially grown on a c-plane sapphire substrate at $1050{ }^{\circ} \mathrm{C}$ with a $25 \mathrm{~nm}$ low-temperature nucleation layer. After that, the template was ramped down to $760{ }^{\circ} \mathrm{C}$ and was deposited with, firstly, a 10 nm-thick GaN layer; secondly, an InGaN layer of nominal thickness and indium composition of 3 $\mathrm{nm}$ and $15 \%$, respectively; lastly, a $10 \mathrm{~nm}$-thick GaN capping layer. The deposition of the $10 \mathrm{~nm}$ low-temperature 
GaN immediately before the QW layer was used to make the SQW structurally symmetric, with both QW barriers grown at $760{ }^{\circ} \mathrm{C}$. All epitaxial processes were carried out using a $3 \times 2$ Thomas-Swan closed-coupled showerhead MOCVD system. After epitaxial growth, arrays of nanoscale chromium $(\mathrm{Cr})$ etch masks with varying diameters are patterned using electron-beam lithography (Raith 150 with MicroChem PMMA A2 resist) and the subsequent lift-off of 20-nm-thick Cr metallization (Enerjet Evaporator). Nanopillars are formed after etching the sample in an inductively coupled plasma reactive-ion etching (ICP-RIE; LAM 9400), using chlorine $\left(\mathrm{Cl}_{2}\right)$ and argon (Ar) as the etch gases. The $\mathrm{Cr}$ mask was removed using the chromium etchant (Cyantek CR-14) for 15 minutes. Immediately after that, 40-nm amorphous $\mathrm{Al}_{2} \mathrm{O}_{3}$ was conformally deposited by atomic layer deposition (ALD, Oxford Instruments) to provide surface passivation and to form a photonic wire structure which facilitates the out-coupling (surfacenormal) of the emission from the InGaN layer [18].

Figure 1(b) shows the scanning electron micrograph (SEM) of a typical InGaN nanopillar array of period 300 $\mathrm{nm}$ after ICP-RIE etching. Across the sample, nanopillars with top diameters ranging from 15 to $30 \mathrm{~nm}$ were made. The inset of Fig. 1(b) shows a single InGaN nanopillar. Its InGaN region has a diameter of $17 \mathrm{~nm}$ and is located at the

(a)
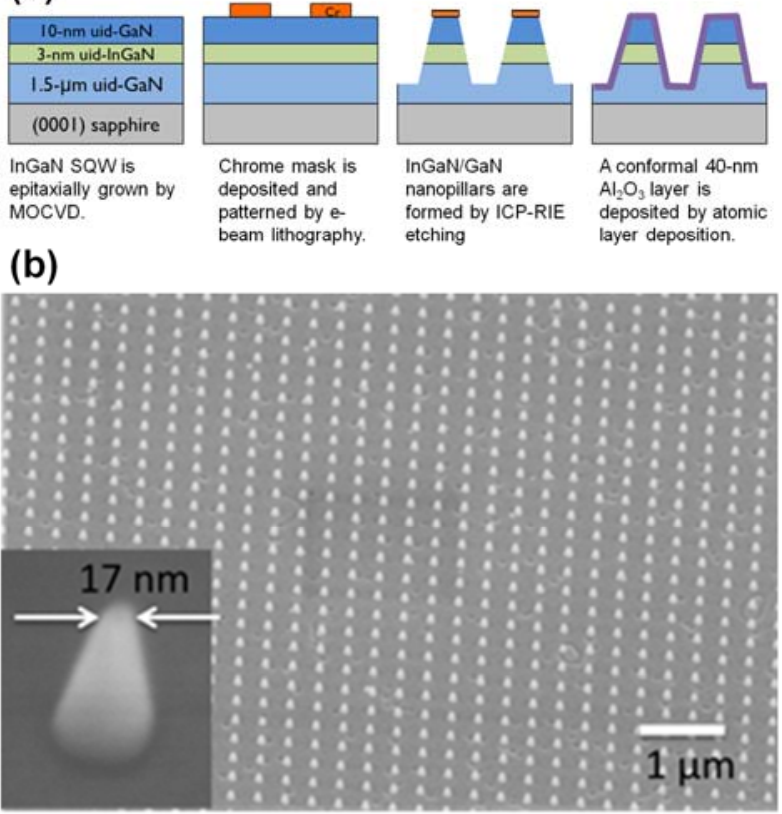

Figure 1 (a) Illustration of the process flow. (b) Scanning electron microscopy (SEM) image of nanopillars before $\mathrm{Al}_{2} \mathrm{O}_{3}$ deposition. The inset shows a standalone single pillar with a diameter of $17 \mathrm{~nm}$ measured at the InGaN plane. (a)

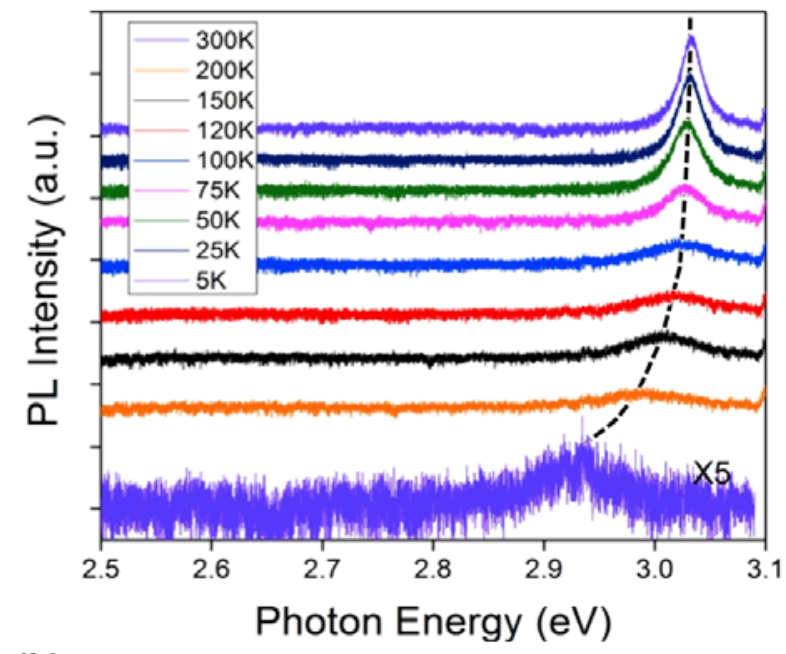

(b)

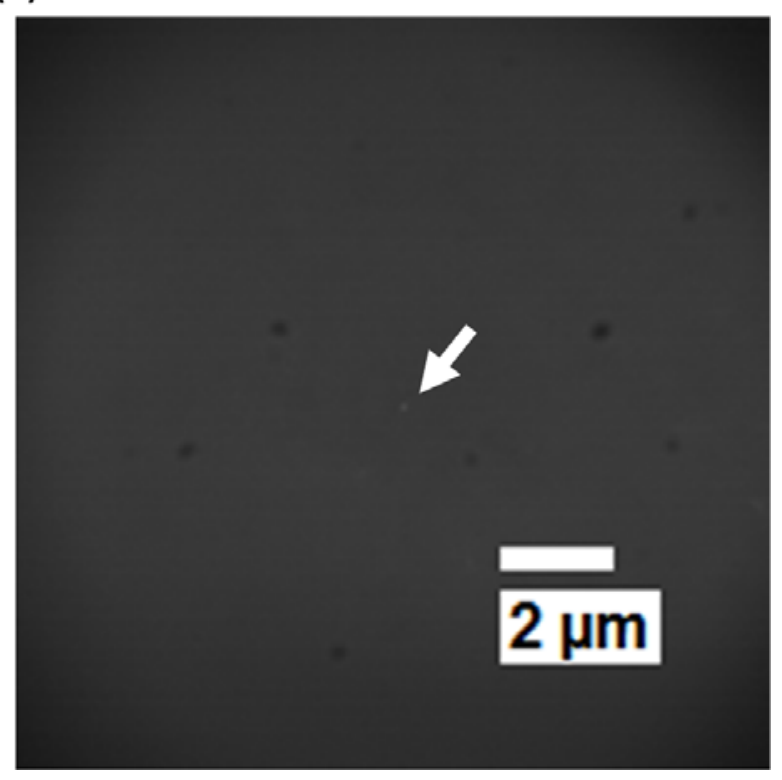

Figure 2 (a) Temperature-dependent PL of a single nanopillar. The dashed line marks the position of the emission peak and shows a trend shifting towards the lower photon energy (redshifted). (b) Real-time CCD image of a single nanopillar at RT.

center of a $100 \times 100 \mu \mathrm{m}^{2}$ area where no other InGaN structures exist. All the pillars are observed to have tapered sidewall of about 80 degree. The tapered sidewall is caused by the mask faceting effect (or the mask erosion) [19], in which the $\mathrm{Cr}$ nanodot etch mask shrinks in diameter due to the physical sputtering of an anisotropic ICP etching. Further evidence for the mask faceting effect comes from the observation that when the Cr nanodots are less than $15 \mathrm{~nm}$, the pillars formed will have diameter larger than $30 \mathrm{~nm}$ and a pillar height much less than the nominal etch height of $120 \mathrm{~nm}$. This implies that the smaller $\mathrm{Cr}$ nanodots are 


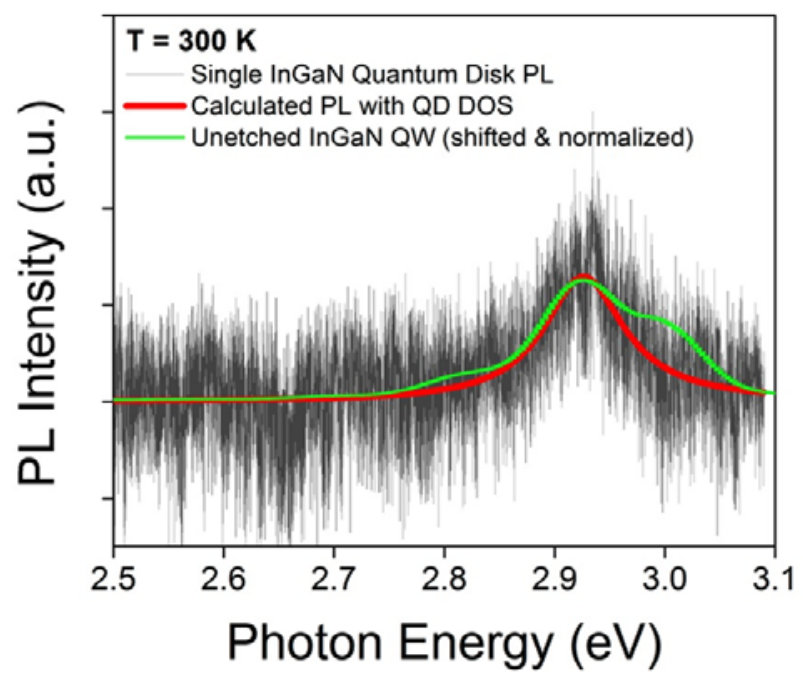

Figure 3 Plots of RT PL of a single nanopillar, an unetched InGaN SQW, and the calculated spontaneous emission spectrum using a 0-D density of states function. The QW PL is shifted in wavelength to make the comparison more convenient.

completely sputtered away during the etching and fail to serve as etch masks, and hence the insufficient etch depth. By patterning $\mathrm{Cr}$ nanodots of graded diameters, the smallest top pillar diameter we can achieve while maintaining the expected etch depth was found to be $13 \mathrm{~nm}$. To further shrink the nanopillar diameter, one must diminish the faceting effect by using a thicker $(>20 \mathrm{~nm}) \mathrm{Cr}$ mask, increasing the pressure during ICP etching, and/or reducing the $\mathrm{Ar}$ to $\mathrm{Cl}_{2}$ ratio to minimize the physical sputterings.

The nanopillar sample was measured using a microphotoluminescence ( $\mu$-PL) setup equipped with a $50 \mathrm{X}$ objective lens, responsible for both excitation and signal collection. The 390-nm ultrafast laser excitation was focused down to a $2-\mu \mathrm{m}$-diameter beam spot, giving an excitation intensity of about $5 \mathrm{~kW} / \mathrm{cm}^{2}$. The PL signal was dispersed by a monochromator with a $50-\mathrm{cm}$ focal length and collected by a photomultiplier tube (PMT). The overall spectral resolution was $1 \AA$.

3 Results and discussion Temperature-dependent PL spectra of the single InGaN nanopillar shown in Fig. 1(b) inset are plotted in Fig. 2(a). First we observe that unlike in the typical InGaN quantum wells, the temperature-dependent PL peak energy follows the Varshni's model $E(T)=E_{0}(T)-\alpha T^{2} /(\beta+T)$ with $\alpha=8.54 \times 10^{-4} \mathrm{eV} / \mathrm{K}$ and $\beta=550 \mathrm{~K}$ instead of the $\mathrm{S}$ shape [20]. At $5 \mathrm{~K}$, the single pillar exhibits a PL peak with a full-width-half-maximum (FWHM) linewidth of $24 \mathrm{meV}$ (3.5 nm in wavelength). This FWHM linewidth is comparable to that of an SK GaN QD in Ref. [3], but is much broader than those from single SK InGaN QDs reported elsewhere [21-24] with only hundreds of $\mu \mathrm{eV}$. We attributed this broad linewidth to the spectral diffusion originated from the large piezoelectric fields present in the III-nitride system [22, 23, 25, 26, 30].

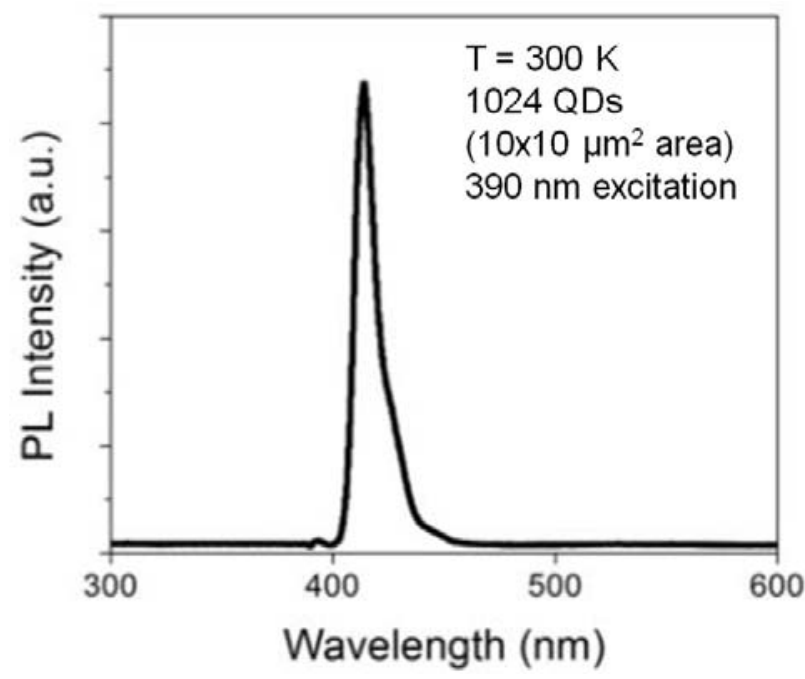

Figure 4 Room-temperature PL of an ensemble of 1024 nanopillars. Laser beam spot size was adjusted to be $10 \times 10 \mu \mathrm{m}^{2}$ so that all 1024 nanopillars were excited.

The trap centers on the etched pillar sidewalls can cause localized fluctuation of electric fields. We observe an increasing broadening of PL linewidth and a red shift of emission peak as the temperature rises. It is worth noting that a stand-alone single pillar exhibits distinct PL signal with a linewidth of $65 \mathrm{meV}$ even at RT, which is different from other reports of self-assembled InGaN QD in which single-dot PL signal drastically diminishes and becomes difficult to detect when temperature rises above $100 \mathrm{~K}$ [2124]. Figure 2(b) shows the real-time CCD image of a single nanopillar under 390-nm laser excitation at RT. The large linewidth at RT is believed to be phonon assisted. From the integrated PL intensity and assuming the radiative efficiency at $5 \mathrm{~K}$ to be $100 \%$, the radiative efficiency of the single nanopillar was found to be $33 \%$ which was attributed to the low surface recombination velocity [27].

We also note that the RT PL spectrum of a single pillar is quite symmetric compared to a typical QW PL in which the asymmetric high-energy tail presents [28]. The highenergy tail originates from the integration of Fermi-Dirac distribution function of the carriers with the twodimensional density of state and is a characteristic of QW PL $[28,29]$. The RT PL signal of a single nanopillar is fitted with the spontaneous emission of QD as described in Ref. [29]. The result of the fitting is shown in Fig. 3. As a comparison, the PL of the unetched SQW sample is also shown (with its peak wavelength shifted for visual convenience). It is evident that the PL from the single InGaN nanopillar fits well with the QD curve but not the QW. We have also observed the size quantization effect, a important characteristic of the quantum dot. The blue shift from a 24$\mathrm{nm}$ disk to a 16-nm disk was measured to be $60 \mathrm{meV}$ and cannot be solely attributed to strain relaxation. The detailed modeling of the size quantization effect will be presented else-where. The above results strongly suggest that the 
disk-shaped InGaN region possess emission characteristics closer to a QD than a QW. Finally, a more rigorous proof of the QD-like emission properties will require the measurement of photon antibunching and is currently underway.

Figure 4 shows the PL from an ensemble of InGaN nanopillars measured at RT. It is seen that the emission linewidth is $63 \mathrm{meV}$ which is the same as that of the single InGaN nanopillar. This implies an extremely well-controlled nanopillar diameters and uniformity. We also observed no obvious yellow band luminescence (YBL) which is an indication of the high materials quality even after the ICP-RIE process. It may be attributed to the conformal coating of $\mathrm{Al}_{2} \mathrm{O}_{3}$ by ALD which passivates the surface. In a separate sample without the $\mathrm{Al}_{2} \mathrm{O}_{3}$ passivation, we indeed observed the formation of YBL over time.

4 Conclusion We demonstrated that the simple topdown approach by dry etching is viable to fabricate highquality site- and dimension-controlled InGaN quantum dots from an InGaN SQW sample using reactive-ion etching. We characterized the emission properties of a single InGaN nanopillar using temperature-dependent microphotoluminescence. Distinct PL signal was observed even at RT which agreed well with the calculated QD emission and was markedly different from the unetched QW PL. The ensemble exhibited a similar emission linewidth as the single nanopillar, indicating the extremely well-controlled nanopillar size and uniformity.

Acknowledgements This work was financially supported by National Science Foundation under grant ECCS \#0901477 and Defense Advanced Research Projects Agency (DARPA) under grant DSO N66001-10-1-4042. The authors would like to thank Professor Hui Deng and Mr. Lei Zhang for fruitful discussions.

\section{References}

[1] Y. Narukawa, Y. Kawakami, S. Fujita, S. Fujita, and S. Nakamura, Phys. Rev. B 55, R1938 (1997).

[2] W. Huang and F. Jain, J. Appl. Phys. 87, 7354 (2000).

[3] S. Kako, C. Santori, K. Hoshino, S. Götzinger, Y. Yamamoto, and Y. Arakawa, Nature Mater. 5, 887 (2006).

[4] G. Khitrova, H. M. Gibbs, M. Kira, S. W. Koch, and A. Scherer, Nature Phys. 2, 81 (2006).

[5] K. Hennessy, A. Badolato, M. Winger, D. Gerace, M. Atatüre, S. Gulde, S. Fält, E. L. Hu, and A. Imamoğlu, Nature 445, 896 (2007).

[6] C. Santori, D. Fattal, J. Vučković, G. S. Solomon, and Y. Yamamoto, Nature 419, 594 (2002).

[7] M. Nomura, N. Kumagai, S. Iwamoto, Y. Ota, and Y. Arakawa, Nature Phys. 6, 279 (2010).

[8] P. Chen, A. Chen, S. J. Chua, and J. N. Tan, Adv. Mater. 19, 1707 (2007).

[9] J. Yamaguchi and K. Okamoto, Jpn. J. Appl. Phys. 32, 1523 (1993).

[10] J. Yamaguchi, J. Ogasawara, and K. Okamoto, J. Appl. Phys. 72, 5919 (1992).

[11] R. Steffen, Th. Koch, J. Oshinowo, F. Faller, and A. Forchel, Appl. Phys. Lett. 68, 223 (1996)
[12] C. Schneider, T. Heindel, A.Huggenberger, P. Weinmann, C. Kristner, M. Kamp, S. Reitzenstein, S. Höfling, and A. Forchel, Appl. Phys. Lett. 94, 111111 (2009).

[13] S. Keller, C. Schaake, N. A. Fichtenbaum, C. J. Neufeld, Y. Wu, K. McGroddy, A. David, S. P. DenBaars, C. Weisbuch, J. S. Speck, and U. K. Mishra, J. Appl. Phys. 100, 054314 (2006).

[14] Y. He, L. Chen, Y.-K. Song, A. V. Nurmikko, S.-R. Jeon, Z. Ren, M. Gherasimova, and J. Han, Phys. Status Solidi C 2, 2740 (2005)

[15] H.-S. Chen, D.-M. Yeh, Y.-C. Lu, C.-Y. Chen, C.-F. Huang, T.-Y. Tang, C.-C. Yang, C.-S. Wu, and C.-D. Chen, Nanotechnology 17, 1454 (2006).

[16] V. Ramesh, A. Kikuchi, K. Kishino, M. Funato, and Y. Kawakami, J. Appl. Phys. 107, 114303 (2010).

[17] P. Yu, C. H. Chiu, Y.-R. Wu, H. H. Yen, J. R. Chen, C. C. Kao, H.-W. Yang, H. C. Kuo, T. C. Lu, W. Y. Yeh, and S. C. Wang, Appl. Phys. Lett. 93, 081110 (2008).

[18] J. Heinrich, A. Huggenberger, T. Heindel, S. Reitzenstein, S. Höfling, L. Worschech, and A. Forchel, Appl. Phys. Lett. 96, 211117 (2010).

[19] M. J. Madou, Fundamentals of Microfabrication: The Science of Miniaturization, 2nd ed. (CRC Press LLC, Boca Raton, 2002), chap. 2.

[20] Y.-H. Cho, G. H. Gainer, A. J. Fischer, J. J. Song, S. Keller, U. K. Michra, and S. P. DenBaars, Appl. Phys. Lett. 73, 1370 (1998).

[21] K. Sebald, H. Lohmeyer, J. Gutowski, T. Yamaguchi, and D. Hommel, Phys. Status Solidi B 243, 1661 (2006).

[22] R. Seguin, S. Rodt, A. Strittmatter, L. Reißmann, T. Bartel, A. Hoffmann, D. Bimberg, E. Hahn, and D. Gerthsen, Appl. Phys. Lett. 84, 4023 (2004).

[23] J. H. Rice, J. W. Robinson, J. D. Smith, A. Jarjour, R. A. Taylor, R. A. Oliver, G. Andrew, D. Briggs, M. J. Kappers, S. Yasin, and C. J. Humphreys, IEEE Trans. Nanotechnol. 3, 343 (2004).

[24] H. Schömig, S. Halm, A. Forchel, G. Bacher, J. Off, and F. Scholz, Phys. Rev. Lett. 92, 106802 (2004).

[25] J. H. Rice, J. W. Robinson, A. Jarjour, R. A. Taylor, R. A. Oliver, G. Andrew, D. Briggs, M. J. Kappers, and C. J. Humphreys, Appl. Phys. Lett. 84, 4110 (2004).

[26] S. A. Empedocles and M. G. Bawendi, Science 278, 2114 (1997).

[27] J. B. Schlager, K. A. Bertness, P. T. Blanchard, L. H. Robins, A. Roshko, and N. A. Sanford, J. Appl. Phys. 103, 124309 (2008).

[28] H. Zhao, G. Liu, and N. Tansu, Appl. Phys. Lett. 97, 131114 (2010).

[29] S. L. Chuang, Physics of Optoelectronic Devices (WileyInterscience, New York, 1995), pp. 157-160, 345-351.

[30] J. H. Rice, J. W. Robinson, J. H. Na, K. H. Lee, R. A. Taylor, D. P. Williams, E. P. O’Reilly, A. D. Andreev, Y. Arakawa, and S. Yasin, Nanotechnology 16, 1477 (2005). 